\title{
Pacific
}

Journal of

Mathematics

\section{BRIDGED EXTREMAL DISTANCE AND MAXIMAL CAPACITY}

ROBERT E. THURMAN 


\title{
BRIDGED EXTREMAL DISTANCE AND MAXIMAL CAPACITY
}

\author{
ROBERT E. THURMAN ${ }^{1}$
}

\begin{abstract}
We develop the concept of "bridged extremal distance" between disjoint sets $X$ and $Z$ on the boundary of a finitely connected domain $G$; that is, the extremal length of the family of curves connecting $X$ and $Z$ which are allowed to stop at a component of the "bridge" $Y=\partial G \backslash(X \cup Z)$ and re-emerge from any other point of that component. We connect bridged extremal distance with the extremal problem of "minimal extremal distance", and express it in terms of the period matrix associated with the harmonic measures of the boundary components of $G$. Then, in direct analogy to Ahlfors and Beurling's extremal length interpretation of logarithmic capacity, we use bridged extremal distance to give an extremal length interpretation of "maximal capacity".
\end{abstract}

\section{Introduction.}

Early in the development of extremal length, Ahlfors and Beurling related it to logarithmic capacity. Their survey article [3] introduced "reduced extremal distance" and connected it to capacity in the following way. Let $\Omega$ be a planar domain containing the point at infinity whose boundary $\Gamma$ consists of a finite number of non-degenerate continua. Then $\Omega$ has a Green's function $g(z)$ with pole at infinity, and

$$
\gamma=\lim _{z \rightarrow \infty}\{g(z)-\log |z|\}
$$

is called Robin's constant for $\Omega$. The logarithmic capacity of $\Gamma$ is $d(\Gamma)=e^{-\gamma}$. To express $\gamma$ in terms of extremal length, consider the domain $\Omega_{R} \subset \Omega$ bounded by $\Gamma$ and the circle $C_{R}$ centered at the origin of radius $R$, where $R$ is large. Let $\lambda_{R}$ denote the extremal distance between $\Gamma$ and $C_{R}$; that is, the extremal length of the family of curves in $\Omega_{R}$ connecting $\Gamma$ to $C_{R}$. Then $\lambda_{R}$ increases to infinity with $R$, and Ahlfors and Beurling (see also [2],

\footnotetext{
${ }^{1}$ This work forms a portion of the author's Ph.D. thesis, and was generously supported in part by a grant from the Alfred P. Sloan Foundation.
} 
Ch. 4, Sec. 14) give the following asymptotic relationship between extremal distance and capacity:

$$
\frac{1}{2 \pi} \gamma=\lim _{R \rightarrow \infty}\left\{\lambda_{R}-\frac{1}{2 \pi} \log R\right\}
$$

The right-hand side is called the reduced extremal distance of $\Gamma$.

In this paper we develop the concept of "bridged extremal distance", and, in direct analogy to (1), relate it to the "maximal capacity" of a boundary subset. We briefly describe both quantities below, so as to state the result more precisely .

Let $f$ be a conformal map of $\Omega$, normalized so that $f(z) \approx z$ near infinity. Then by conformal invariance of Green's function, $f$ preserves the capacity (and hence the reduced extremal distance) of the full boundary $\Gamma$. On the other hand, if $A$ is a subset of $\Gamma$ consisting of some number of components of $\Gamma$, then the capacity of $A$ is likely to change under such maps. This leads us to consider the maximal capacity of $A$,

$$
d_{m}(A)=\sup _{f} d(f(A))
$$

where the supremum ranges over all normalized conformal maps $f$ of $\Omega$. It was shown in [14] that extremal functions always exist, and the maximal capacity was expressed in terms of the capacity of the full boundary and the harmonic measures of each of the components of $\Gamma \backslash A$. The results are restated as Theorem B in Section 2 of this paper.

Bridged extremal distance is defined as follows. Fix an arbitrary finitelyconnected domain $G$, and partition its boundary $S$ into subsets $X, Y$, and $Z$, each consisting of a union of components of $S$. Consider the family $\Phi_{b}$ of rectifiable curves in $G$ connecting $X$ to $Z$, using $Y$ as a "bridge" (see Figure 1 ). That is, in connecting $X$ to $Z$, a curve in this bridging family is allowed to stop at a point of a component of $Y$ and re-emerge at any other point of that same component. Define the bridged extremal distance $\lambda^{b}=\lambda_{G}^{b}(X, Y, Z)$ to be the extremal length of $\Phi_{b}$, according to the precise definition of extremal length in Section 2.

A main goal of this paper is to establish the following connection between bridged extremal distance and maximal capacity. With $\Omega$ as before, let $B$ be the set $\Gamma \backslash A$. Again form $\Omega_{R} \subset \Omega$, and compute the bridged extremal distance between $A$ and $C_{R}$, using $B$ as the bridge:

$$
\lambda_{R}^{b}=\lambda_{\Omega_{R}}^{b}\left(A, B, C_{R}\right)
$$




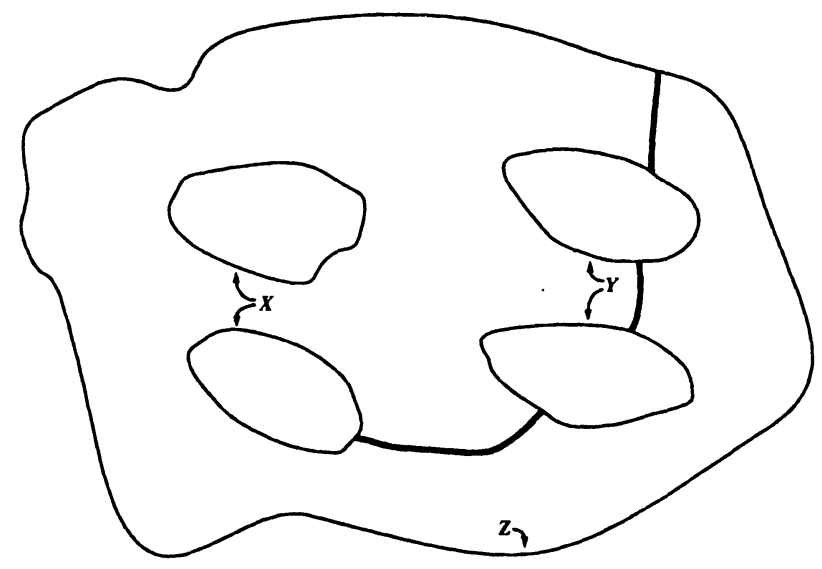

Figure 1. A curve in $\Phi_{b}$.

Then with $\gamma_{m}$ defined by $d_{m}(A)=\exp \left(-\gamma_{m}\right)$, we prove that

$$
\frac{1}{2 \pi} \gamma_{m}=\lim _{R \rightarrow \infty}\left\{\lambda_{R}^{b}-\frac{1}{2 \pi} \log R\right\}
$$

This is the content of Theorem 4.

The proof hinges on the relation of bridged extremal distance to the solution of another extremal problem. Consider any conformal map $f$ of the domain $G$, and define $\widehat{f(G)}$ to be the domain containing $f(G)$ which is bounded only by $f(X)$ and $f(Z)$; that is, obtain $\widehat{f(G)}$ from $f(G)$ by filling in the holes made by $f(Y)$. Let $\hat{\lambda}_{f}$ denote the extremal distance between $f(X)$ and $f(Z)$ relative to this larger domain. Then $\widehat{\lambda}_{f}$ depends on the conformal map $f$, so we define the minimal extremal distance between $X$ and $Z$ in $G$ to be

$$
\widehat{\lambda}=\hat{\lambda}_{G}(X, Y, Z)=\inf _{f} \hat{\lambda}_{f}
$$

with the infimum taken over all conformal maps of $G$. In Section 3 we use a variational method to prove Theorem 1 , which expresses $\hat{\lambda}$ in terms of the harmonic measures of the components of $S$ and establishes the existence of extremal functions. In Section 4 we show that bridged extremal distance equals minimal extremal distance: $\lambda^{b}=\widehat{\lambda}$. This is Theorem 2. The proof of Theorem 4 then proceeds in Section 5, using some special properties of minimal extremal distance.

In [6] Duren and Schiffer considered the minimal capacity of a boundary subset,

$$
\inf _{f} d(f(A))
$$


where the infimum is taken over all normalized conformal maps of $\Omega$. They showed that the minimal capacity of $A$ equals the "Robin capacity" of $A$. Duren and Pfaltzgraff [5] then exploited an extremal length interpretation of Robin capacity. We compare our maximal capacity results with those of Duren and Pfaltzgraff in Section 6. In Section 7 we show how minimal and bridged extremal distances can be interpreted for more general partitions $X \cup Y \cup Z$ of the boundary $S$.

Note. After this paper had been prepared for publication, the author learned that families of bridging curves on Riemann surfaces were considered in the 1970's by David Minda [8, 9]. He generalized earlier results on the same subject by Marden and Rodin [7]. We briefly compare their results with ours in the final section. We thank David Minda for calling our attention to this earlier work.

\section{Background.}

We begin by reviewing the definitions and some of the key results in the method of extremal length, and then state the main result on maximal capacity from [14]. See Ahlfors [2] (Chapter 4) or Ohtsuka [12] for a detailed discussion of the following material.

Let $\Phi$ be a family of rectifiable curves lying in a domain $U$. Call a nonnegative Borel-measurable function $\rho(x, y)$ defined in $U$ an admissible metric for $\Phi$ if

$$
\int_{\phi} \rho d s \geq 1
$$

for all curves $\phi \in \Phi$. The left-hand side is called the $\rho$-length of $\phi$. Define the modulus of $\Phi$ to be

$$
M(\Phi)=\inf \left\{\iint_{U} \rho^{2} d x d y: \quad \rho \text { is admissible }\right\} .
$$

Define the extremal length $\lambda(\Phi)$ to be $1 / M(\Phi)$. If there are no admissible metrics, set $M(\Phi)=\infty$ and $\lambda(\Phi)=0$. Call $\rho_{0}$ an extremal metric if

$$
M(\Phi)=\iint_{U} \rho_{0}^{2} d x d y
$$

Two simple inequalities follow immediately from the definition.

Comparison Principles. Let $\Phi_{1}$ and $\Phi_{2}$ be families of curves.

(i) If $\Phi_{1} \subseteq \Phi_{2}$ then $\lambda\left(\Phi_{2}\right) \leq \lambda\left(\Phi_{1}\right)$.

(ii) If every curve in $\Phi_{1}$ has a subcurve in $\Phi_{2}$, then $\lambda\left(\Phi_{2}\right) \leq \lambda\left(\Phi_{1}\right)$. 
There is a physical interpretation of extremal length. Think of the curve family $\Phi$ as representing a system of homogeneous electric wires. Then the extremal length $\lambda(\Phi)$ represents the resistance of $\Phi$. The above lemmas reflect the fact that systems of fewer, longer wires have greater resistance.

A conformal map $f$ of $U$ transforms the family $\Phi$ into a family $f(\Phi)$ lying in the domain $f(U)$. The map provides a one-to-one correspondence between admissible metrics in the two domains, and conformal invariance of extremal length follows esily:

$$
\lambda(f(\Phi))=\lambda(\Phi) .
$$

In this paper we will primarily consider the family of curves connecting disjoint sets $E_{1}$ and $E_{2}$ lying on the boundary of a domain $U$. The extremal length of this family is called the extremal distance between $E_{1}$ and $E_{2}$ in $U$, denoted $\lambda_{U}\left(E_{1}, E_{2}\right)$. The following theorem [2] shows that in the case of extremal distance, extremal metrics can be expressed in terms of a generalization of harmonic measure.

Theorem A. Let $E_{1}$ and $E_{2}$ be disjoint sets lying on $\partial U$, each a finite union of arcs or closed curves. Let $\omega$ be the harmonic function satisfying the following boundary conditions:

(i) $\omega=0$ on $E_{1}$.

(ii) $\omega=1$ on $E_{2}$.

(iii) $\frac{\partial \omega}{\partial n}=0$ on $\partial U \backslash\left(E_{1} \cup E_{2}\right)$.

Then $\rho_{0}=|\nabla \omega|$ is the extremal metric for $\lambda_{U}\left(E_{1}, E_{2}\right)$. That is,

$$
\lambda_{U}\left(E_{1}, E_{2}\right)=\left[D_{U}(\omega)\right]^{-1},
$$

where

$$
D_{U}(\omega)=\iint_{U}\left(\omega_{x}^{2}+\omega_{y}^{2}\right) d x d y
$$

is the Dirichlet integral of $\omega$ in $U$.

By conformal invariance, we may assume $\partial U$ is a union of analytic curves, so that the boundary conditions on $\omega$ make sense. We will not give the proof of Theorem A here, but it is easy enough (and will suit our purposes later) to show that $\rho_{0}=|\nabla \omega|$ is an admissible metric. For this, let $\phi$ be a rectifiable curve in $U$ connecting $E_{1}$ to $E_{2}$. Then

$$
\int_{\phi} \rho_{0} d s=\int_{\phi}|\nabla \omega||d z| \geq \int_{\phi}|\nabla \omega \cdot d z|=\left|\int_{\phi} d \omega\right|=1 .
$$

Note that the "flow-lines" of $\omega$ (the curves tangent to $\nabla \omega$ ) have $\rho_{0}$-length exactly equal to 1 , so provide the shortest paths from $E_{1}$ to $E_{2}$ in this metric. 
Theorem A provides the key to relation (1) between extremal length and capacity. Roughly speaking, since Green's function $g$ of $\Omega$ is identically zero on $\Gamma$, and since $g(z) \approx \log R+\gamma$ on the circle $C_{R}$ for large $R$,

$$
\frac{g(z)}{\log R+\gamma}
$$

approximates the harmonic measure $\omega_{R}$ of $C_{R}$ relative to $\Omega_{R}$. If we assume the approximation is exact, then Theorem A says

$$
\frac{1}{\lambda_{R}}=D_{\Omega_{R}}\left(\frac{g(z)}{\log R+\gamma}\right) \text {. }
$$

Green's theorem then shows that the right-hand side equals $2 \pi /\{\log R+\gamma\}$. Thus

$$
\frac{1}{2 \pi} \gamma=\lambda_{R}-\frac{1}{2 \pi} \log R
$$

under our assumption about $g$.

We now restate the main theorem on maximal capacity from [14]. Let $\Omega$ be a domain containing $\infty$ whose boundary $\Gamma$ consists of a finite union of nondegenerate continua. Let $\Gamma$ be partitioned into subsets $A$ and $B$, each a union of boundary components:

$$
A=A_{1} \cup \cdots \cup A_{k}, \quad B=B_{1} \cup \cdots \cup B_{m} .
$$

Let $h_{i}(z)$ be the harmonic measure of $B_{i}$ with respect to $\Omega$, and set $v_{i}=$ $h_{i}(\infty)$ for $i=1, \ldots, m$. Let $p_{i j}$ be the period of the harmonic conjugate of $h_{i}$ about $B_{j}$ for $i, j=1, \ldots, m$ :

$$
p_{i j}=\frac{1}{2 \pi} \int_{L} \frac{\partial h_{i}}{\partial n} d s
$$

where $L$ is any positively oriented analytic closed curve looping around $B_{\jmath}$ exactly once, and not enclosing any other boundary component. Set $\mathbf{v}=$ $\left(v_{1}, \ldots, v_{m}\right)$ and $\mathbf{P}=\left(p_{i \jmath}\right)$. For any normalized conformal map $f$ of $\Omega$, denote by $\widehat{f(\Omega)}$ the domain containing $f(\Omega)$ which is exterior to $f(A)$. Let $\widehat{g}(z)$ denote Green's function with pole at infinity for $\widehat{f(\Omega)}$. Recall that the maximal capacity $d_{m}(A)$ of $A$ is the supremum of the capacities $d(f(A))$ as $f$ ranges over all normalized conformal maps of $\Omega$.

Theorem B. With the above definitions,

$$
d_{m}(A)=d(\Gamma) \exp \left\{-\sum_{i=1}^{m} c_{i} v_{i}\right\}
$$


where $\mathbf{c}=\left(c_{1}, \ldots, c_{m}\right)$ and

$$
\mathbf{c}^{T}=\mathbf{P}^{-1} \mathbf{v}^{T}
$$

Extremal functions $F$ satisfying $d(F(A))=d_{m}(A)$ always exist. Extremal domains $F(\Omega)$ are characterized by the property that each component $F\left(B_{\imath}\right)$ of $F(B)$ is a union of analytic arcs on which $\widehat{g}$ is constant. That is, $d(F(A))=$ $d_{m}(A)$ if and only if $\hat{g}(z)$ is constant on $F\left(B_{i}\right)$, for each $i=1, \ldots, m$. In this extremal case, the constant values are uniquely determined and are given by the vector $\mathbf{c}: \widehat{g}(z)=c_{i}$ on $F\left(B_{i}\right)$.

Corollary. Let $\gamma_{m}$ be such that $d_{m}(A)=e^{-\gamma_{m}}$. Let $g(z)$ be Green's function for $\Omega$ with pole at infinity, and let $h_{i}(z)$ be the harmonic measure of $B_{i}$ with respect to $\Omega$. Set $M(z)=g(z)+\sum_{i=1}^{m} c_{i} h_{i}(z)$, where $c_{i}$ is defined in (3). Then $\gamma_{m}=\lim _{z \rightarrow \infty}\{M(z)-\log |z|\}$.

\section{Minimal extremal distance.}

We now give an explicit expression for minimal extremal distance and characterize extremal domains in a way that parallels Theorem B. The crux of both proofs is an application of Schiffer's method of boundary variation to extremal domains.

Let $G$ be a domain whose boundary $S$ consists of a finite union of nondegenerate continua. Let $S$ be partitioned into subsets $X, Y$ and $Z$, each a union of boundary components:

$$
\begin{aligned}
& X=X_{1} \cup \cdots \cup X_{r}, \\
& Y=Y_{1} \cup \cdots \cup Y_{s}, \\
& Z=Z_{1} \cup \cdots \cup Z_{t} .
\end{aligned}
$$

Let $\eta_{Z}(z)$ be the harmonic measure of $Z$ relative to $G$ and let $\eta_{i}(z)$ be the harmonic measure of $Y_{i}$, for $i=1, \ldots, s$. Let $w_{i}$ and $w_{Z}$ be the periods of the harmonic conjugate of $\eta_{i}$ and $\eta_{Z}$, respectively, about $Z$. Let $q_{i j}$ be the period of the harmonic conjugate of $\eta_{i}$ about $Y_{j}$. Set $\mathbf{w}=\left(-w_{1}, \ldots,-w_{s}\right)$ and $\mathbf{Q}=\left(q_{i j}\right)$. For any conformal map $f$ of $G$, denote by $\widehat{f(G)}$ the domain containing $f(G)$ which is bounded only by $f(X)$ and $f(Z)$. Let $\widehat{\eta}_{Z}(\zeta)$ denote the harmonic measure of $f(Z)$ relative to $\widehat{f(G)}$. Recall that $\hat{\lambda}_{f}$ denotes the extremal distance between $f(X)$ and $f(Z)$ in $\widehat{f(G)}$, and that the minimal extremal distance $\hat{\lambda}$ between $X$ and $Z$ in $G$ is the infimum of $\hat{\lambda}_{f}$ over all 
conformal maps $f$ of $G$.

Theorem 1. With the above definitions,

$$
\hat{\lambda}=\left[2 \pi\left(w_{Z}+\sum_{i=1}^{s} b_{i} w_{i}\right)\right]^{-1},
$$

where $\mathbf{b}=\left(b_{1}, \ldots, b_{s}\right)$ and

$$
\mathbf{b}^{T}=\mathbf{Q}^{-1} \mathbf{w}^{T}
$$

Extremal functions $F$ satisfying $\widehat{\lambda}_{F}=\widehat{\lambda}$ always exist. Extremal domains $F(G)$ are characterized by the property that each component $F\left(Y_{i}\right)$ of $F(Y)$ is a union of analytic arcs on which $\widehat{\eta}_{Z}$ is constant. That is, $\widehat{\lambda}_{F}=\hat{\lambda}$ if and only if $\widehat{\eta}_{Z}$ is constant on $F\left(Y_{i}\right)$, for each $i=1, \ldots, s$. In this extremal case, the constants are uniquely determined and are given by the vector $\mathbf{b}: \widehat{\eta}_{Z} \equiv b_{i}$ on $F\left(Y_{i}\right)$.

Proof. We begin with a compactness argument to establish the existence of extremal maps. Let $\left\{f_{n}\right\}$ be a sequence of conformal maps of $G$ such that $\hat{\lambda}_{f_{n}} \rightarrow \hat{\lambda}$. Normalize the functions so that $f_{n}\left(z_{1}\right)=0$ and $f_{n}^{\prime}\left(z_{1}\right)=1$ for each $n$, where $z_{1}$ is an arbitrary fixed point of $G$. The normalization will not change the value of $\hat{\lambda}_{f_{n}}$, but will make the sequence normal and compact. Thus we may assume that $\left\{f_{n}\right\}$ converges locally uniformly in $G$ to a conformal map $F$. By the Carathéodory convergence theorem, the domains $f_{n}(G)$ converge to their kernel, which is $F(G)$. Thus the domains $\widehat{f_{n}(G)}$ converge to $\widehat{F(G)}$. A theorem of Navoyan [10], modified slightly to hold in the complex plane, guarantees the convergence of the sequence $\left\{\widehat{\lambda}_{f_{n}}\right\}$ to the extremal distance $\hat{\lambda}_{F}$. Thus $\hat{\lambda}_{F}=\hat{\lambda}$.

The next step is to apply a boundary variation to obtain information about extremal domains. We assume from the beginning that $G$ is extremal. Then by Theorem A and Green's Theorem,

$$
\frac{1}{\widehat{\lambda}}=D_{\widehat{G}}\left(\widehat{\eta}_{Z}\right)=\int_{Z} \frac{\partial \widehat{\eta}_{Z}}{\partial n} d s=2 \pi \widehat{p}
$$

where $n$ denotes the direction of the outward pointing normal, and $\widehat{p}$ is the period of the harmonic conjugate of $\widehat{\eta}_{Z}$ about $Z$.

For each fixed point $z_{0}$ in $Y$, there is a family of functions

$$
V_{\rho}(z)=z+\frac{a \rho^{2}}{z-z_{0}}+O\left(\rho^{3}\right), \quad \rho \geq 0
$$


analytic and univalent in the complement of some small connected subset of $Y$ containing $z_{0}$ (see Duren [4], Section 10.3). Let $G^{*}=V_{\rho}(G)$, and let $\widehat{G}^{*} \supset G^{*}$ be the domain bounded only by $X^{*}=V_{\rho}(X)$ and $Z^{*}=V_{\rho}(Z)$. As above, the extremal distance of $X^{*}$ and $Z^{*}$ in $\widehat{G}^{*}$ is

$$
\frac{1}{\lambda^{*}}=2 \pi p^{*}
$$

where $p^{*}$ is the period of the harmonic conjugate of the harmonic measure of $Z^{*}$ relative to $\widehat{G}^{*}$. Since $G$ is extremal for $\hat{\lambda}$,

$$
p^{*} \leq \widehat{p}
$$

Each of the boundary variations $V_{\rho}(z)$ of $G$ acts also as an interior variation of the domain $\widehat{G}$. A variational formula due to Schiffer [13] allows us to relate the two periods:

$$
p^{*}=\widehat{p}+\operatorname{Re}\left\{-a \rho^{2} H_{Z}^{\prime}\left(z_{o}\right)^{2}\right\}+O\left(\rho^{3}\right),
$$

where $H_{Z}$ is the analytic completion of $\widehat{\eta}_{Z}$. Thus by (7),

$$
\operatorname{Re}\left\{-a \rho^{2} H_{Z}^{\prime}\left(z_{o}\right)^{2}\right\}+O\left(\rho^{3}\right) \leq 0 .
$$

We may now invoke Schiffer's theorem for the method of boundary variation ([4], Section 10.3). Since (8) holds for each $z_{0} \in Y$, and since $H_{Z}^{\prime}(z)$ is analytic and not identically zero on $Y$, the theorem says that $Y$ must be the union of analytic arcs lying on the trajectories of the quadratic differential

$$
H_{Z}^{\prime}(z)^{2} d z^{2}<0 \text {. }
$$

Parametrizing each component $Y_{i}$ of $Y$ by $z=z(t)$, we conclude that

$$
\frac{d}{d t} H_{Z}(z(t))=H_{Z}^{\prime}(z(t)) z^{\prime}(t)
$$

is purely imaginary, or that

$$
\frac{d}{d t} \widehat{\eta}_{Z}(z(t))=\frac{d}{d t} \operatorname{Re}\left\{H_{Z}(z(t))\right\}=0
$$

on $Y$. In other words, $\widehat{\eta}_{Z}(z) \equiv b_{i}$ on $Y_{i}$ for some constants $b_{i}$ with $0<b_{i}<1$, for $i=1, \ldots, s$.

We next compute the constants $b_{i}$. Since $\widehat{\eta}_{Z}(z) \equiv b_{i}$ on $Y_{i}$,

$$
\widehat{\eta}_{Z}(z)=\eta_{Z}(z)+\sum_{i=1}^{s} b_{i} \eta_{i}(z)
$$


in $G$. Fix $j \in\{1, \ldots, s\}$. Since $Y_{j}$ is a union of analytic arcs, the normal direction is defined almost everywhere. Therefore, we may integrate the derivative with respect to the outer normal of both sides of (9) about the curve $Y_{j}$ :

$$
\int_{Y_{j}} \frac{\partial \widehat{\eta}_{Z}}{\partial n} d s=\int_{Y_{j}} \frac{\partial \eta_{Z}}{\partial n} d s+\sum_{i=1}^{s} b_{i} \int_{Y_{j}} \frac{\partial \eta_{i}}{\partial n} d s
$$

By definition,

$$
\int_{Y_{j}} \frac{\partial \eta_{i}}{\partial n} d s=2 \pi q_{i j}
$$

And by the symmetry of the period matrix,

$$
\int_{Y_{J}} \frac{\partial \eta_{Z}}{\partial n} d s=2 \pi w_{j}
$$

Since $\widehat{\eta}_{Z}$ is harmonic on and around each $Y_{j}$,

$$
\int_{Y_{j}} \frac{\partial \widehat{\eta}_{Z}}{\partial n} d s=0
$$

Thus, (10) reduces to the equation

$$
-w_{j}=\sum_{i=1}^{s} b_{i} q_{i j}
$$

Repeating this calculation for each $j \in\{1, \ldots, s\}$, we find that

$$
\mathrm{Qb}^{T}=\mathbf{w}^{T} \text {. }
$$

But $\mathbf{Q}$ is invertible. To see this, recall that for the $m \times m$ period matrix $\mathbf{R}=$ $\left(r_{i j}\right)$ of an arbitrary m-connected domain $D$, the $(m-1) \times(m-1)$ submatrix $\mathbf{R}^{\prime}=\left(r_{i j}\right)$, for $i, j=1, \ldots, m-1$ is positive-definite, hence invertible. (See, for instance, Nehari [11], Ch. I, Sect. 10.) The invertibility of $\mathbf{Q}$ follows, since any square submatrix centered on the diagonal of a positive-definite matrix is also positive-definite.

We now compute $\hat{\lambda}=\widehat{\lambda}_{F}$. Equations (6) and (9) yield

$$
\frac{1}{\hat{\lambda}}=\int_{Z} \frac{\partial \widehat{\eta}_{Z}}{\partial n} d s=\int_{Z} \frac{\partial}{\partial n}\left(\eta_{Z}+\sum_{i=1}^{s} b_{i} \eta_{i}\right) d s .
$$


The last integral reduces to

$$
2 \pi\left(w_{Z}+\sum_{i=1}^{s} b_{i} w_{i}\right)
$$

proving (4).

Finally, observe that none of the previous calculations depend on the extremal character of the domain. The fact that $\hat{\eta}_{Z}$ is constant on each $Y_{i}$ completely determines the $b_{i}$ and the extremal distance $\widehat{\lambda}_{F}$, which is thus minimal. In other words, the extremal domains are characterized by the fact that $\widehat{\eta}_{Z}$ is constant on each $Y_{i}$. This completes the proof of the theorem.

Example. Suppose $X$ and $Z$ are both single components of the boundary $S$. Then $G$ can be mapped conformally to an annulus with concentric circular slits such that $X$ and $Z$ correspond to the inner and outer circles, respectively, and each $Y_{i}$ corresponds to one of the slits. If the inner and outer radii equal 1 and $R$, the map $F$ is unique up to rotation. (See Ahlfors [1], Chapter 6, Section 5.1, for example.) Then $\log |z| / \log R$ is the harmonic measure $\widehat{\eta}_{Z}$ of the full annulus $\widehat{F(G)}$, and is constant on each of the slits. By Theorem 1 , this is a configuration minimizing the extremal distance between $X$ and $Y$. The extremal distance is easily computed to be $\frac{1}{2 \pi} \log R$.

Any doubly-connected domain can be mapped conformally to an annulus. The ratio of the inner and outer radii of any such annulus is a conformal invariant, called the modulus of the doubly-connected domain. Thus for any conformal map $f$ of the domain $G$, the modulus of the doubly-connected domain bounded only by $f(X)$ and $f(Z)$ is a minimum (and equal to $R$ ) when $f(G)$ is an annulus minus concentric circular slits.

This example provides us with a geometric interpretation of the constants $b_{i}$ in this special case. Since $\widehat{\eta}_{Z}=\log |z| / \log R$, Theorem 1 says that $b_{i}=$ $\log r_{i} / \log R$, where $r_{i}$ is the radius of the circular slit corresponding to $Y_{i}$.

Let $v(z)=\eta_{Z}(z)+\sum_{i=1}^{s} b_{i} \eta_{i}(z)$. From (10) and (11) we see that the period of the harmonic conjugate of $v$ about $Y_{i}$ is 0 for $i=1, \ldots, s$. And from (6), the period about $Z$ equals $(2 \pi \hat{\lambda})^{-1}$, or $(\log R)^{-1}$. Thus the conjugate of $(\log R) v$ has period 1 about $Z$, periods 0 about $Y_{i}$ for $i=1, \ldots, s$, and consequently period -1 about $X$. Let $f(z)$ be the (multiple-valued) analytic function whose real part is $(\log R) v(z)$. Then it is known (see Ahlfors again) that the above period values guarantee that $e^{f(z)}$ is, up to a rotation, the unique conformal map of $G$ onto a slit annulus of inner radius 1 . 


\section{Minimal extremal distance $=$ bridged extremal distance.}

Recall that the bridged extremal distance is the extremal length of the family $\Phi_{b}$ of curves in $G$ bridging $Y$ in connecting $X$ to $Z$. This definition is conformally invariant in the sense that, for any conformal map $f$ of $G$, the family of curves bridging $f(Y)$ in connecting $f(X)$ to $f(Z)$ is just $f\left(\Phi_{b}\right)$. For a fixed such map, every curve $\widehat{\phi}$ in the family $\widehat{\Phi}_{f}$ of curves connecting $f(X)$ and $f(Z)$ in the domain $\widehat{f(G)} \supset f(G)$ has a subcurve in the bridging family $f\left(\Phi_{b}\right)$. By the comparison principle, we immediately have

$$
\lambda^{b}=\lambda\left(f\left(\Phi_{b}\right)\right) \leq \hat{\lambda}_{f}
$$

whence $\lambda^{b} \leq \hat{\lambda}$. Thus the equality $\lambda^{b}=\hat{\lambda}$ given by Theorem 2 below means that there is some conformal map $F$ of $G$ which collapses $Y$ in such a way that there is no advantage in using $F(Y)$ as a bridge in connecting $F(X)$ to $F(Z)$. The map $F$ must also minimize the extremal distance between $F(X)$ and $F(Z)$. The preceding example illustrates the situation. A curve connecting the inner and outer radii of the annulus makes progress only by moving in a radial direction. It gains no advantage by stopping at one of the concentric slits and continuing on from a different point of that slit.

Theorem 2. Let $G, X, Y$, and $Z$ be as in Theorem 1, and let $\lambda^{b}$ and $\hat{\lambda}$ denote the bridged extremal distance and minimal extremal distance, respectively. Then

$$
\lambda^{b}=\hat{\lambda}
$$

The extremal metric for $\lambda^{b}$ is $\rho_{0}=|\nabla u|$, where

$$
u(z)=\eta_{Z}(z)+\sum_{i=1}^{s} b_{i} \eta_{i}(z)
$$

and $\eta_{Z}, \eta_{i}$, and $b_{i}$ are also as in Theorem 1.

Proof. By the above remarks, we need only show $\lambda^{b} \geq \hat{\lambda}$. Consider the metric $\rho_{0}=|\nabla u|$. If $\phi \in \Phi_{b}$, then

$$
\int_{\phi} \rho_{0} d s \geq\left|\int_{\phi} d u\right|
$$

just as in (2). The curve $\phi$ may make jumps on components of $Y$. But since $u$ is constant on each of those components, the total change in $u$ on $\phi$. is just the difference between the values of $u$ on $X$ and $Z$, or 1 :

$$
\left|\int_{\phi} d u\right|=1
$$


Thus $\rho_{0}$ is admissible for the bridging family $\Phi_{b}$, so

$$
\frac{1}{\lambda^{b}}=M\left(\Phi_{b}\right) \leq \iint_{G} \rho_{0}^{2}=D_{G}(u)
$$

But

$$
D_{G}(u)=\frac{1}{\hat{\lambda}}
$$

by the proof of Theorem 1 , so it follows that $\lambda^{b} \geq \hat{\lambda}$.

With a little extra work, the proofs of Theorems 1 and 2 lead to three different characterizations of the constants $b_{i}$.

Theorem 3. Let $\eta_{Z}$ and $\eta_{i}$ denote the harmonic measures of $Z$ and $Y_{i}$, respectively, relative to the domain $G$. For any conformal map $f$ of $G$, let $\widehat{\eta}_{Z}$ denote the harmonic measure of $f(Z)$ relative to the domain $\widehat{f(G)} \supset f(G)$ bounded only by $f(X)$ and $f(Z)$. Let $v(z)=\eta_{Z}(z)+\sum_{i=1}^{s} a_{i} \eta_{i}(z)$, where $a_{\imath} \in \mathbf{R}$. Then

(i) $\widehat{\eta}_{Z}$ is constant on each $f\left(Y_{i}\right)$ if and only if $\widehat{\eta}_{Z}=b_{i}$ on $f\left(Y_{i}\right)$ for $i=$ $1, \ldots, s$.

(ii) The period of the harmonic conjugate of $v$ about $Y_{i}$ is zero for $i=$ $1, \ldots, s$ if and only if $a_{i}=b_{i}$ for each $i$.

(iii) The Dirichlet integral $D_{G}(v)$ as a function of the $a_{i}$ is a minimum if and only if $a_{i}=b_{i}$.

Proof. The first assertion is contained in Theorem 1. The calculations spanned by (10) and (13) in the proof of Theorem 1 verify assertion (ii). For statement (iii), note that the same reasoning applied in the proof of Theorem 2 shows that $\rho=|\nabla v|$ is an admissible metric for $\Phi_{b}$, simply because $v$ is constant on each $Y_{i}$. Thus $D_{G}(v) \geq M\left(\Phi_{b}\right)$, with equality for $a_{i}=b_{i}$. To see that this is the only possibility, expand $D_{G}(v)$ via Green's Theorem to see that it is a quadratic function of the $a_{i}$, and so can have only one absolute minimum. Indeed, if we set the gradient of this function of the $a_{i}$ equal to zero, we are led directly to equations (12) and (13).

\section{Bridged extremal distance and maximal capacity.}

We turn now to the relation between bridged extremal distance and maximal capacity, expressed in Theorem 4 below. Following the proof, we discuss the connection between the conformally invariant constants defining bridged extremal distance and those defining maximal capacity. The results are contained in Theorem 5 . We conclude the section with an example illustrating both theorems. 
Recall that $\Omega_{R}$ is the domain contained in $\Omega$ which is bounded by $\Gamma$ and the circle $C_{R}$ centered at the origin, of radius $R$, where $R$ is large enough so that $C_{R}$ surrounds $\Gamma$. Define $\lambda_{R}^{b}$ to be the bridged extremal distance between $A$ and $C_{R}$ in $\Omega_{R}$, using $B$ as the bridge.

Theorem 4. Let $\Omega$ be a domain containing the point at infinity whose boundary $\Gamma$ consists of a finite number of non-degenerate continua. Let $\Gamma$ be partitioned into subsets $A$ and $B$, each a union of components of $\Gamma$. Then with the bridged extremal distance $\lambda_{R}^{b}$ defined as above,

$$
\frac{1}{2 \pi} \gamma_{m}=\lim _{R \rightarrow \infty}\left\{\lambda_{R}^{b}-\frac{1}{2 \pi} \log R\right\}
$$

where the maximal capacity $d_{m}(A)$ equals $e^{-\gamma_{m}}$.

The proof is rather technical, so it may be helpful to begin with a sketch of the main ideas. By Theorem 2, the bridged extremal distance $\lambda_{R}^{b}$ equals the minimal extremal distance between $A$ and $C_{R}$ in $\Omega_{R}$. It is the properties of minimal extremal distance which will lead us to (15). We show that the normalized conformal map of $\Omega$ maximizing the capacity of $A$ essentially minimizes the extremal distance between $A$ and $C_{R}$, for large $R$. Intuitively, this makes some sense. To attain the maximal capacity, $A$ must "expand" as much as possible. But when $A$ expands, the "distance" between $A$ and $C_{R}$ decreases. A rigorous argument follows from the characterization of extremal domains for maximal capacity and minimal extremal distance. To highlight the argument, assume that $\Omega$ is extremal for maximizing the capacity of $A$. Then by Theorem B, Green's function $\widehat{g}$ for $\widehat{\Omega}$ is constant on $B$. Moreover, $\widehat{g} \approx \log R+\gamma_{m}$ on $C_{R}$. So as in the comments following Theorem $\mathrm{A}$ in Section 2,

$$
\frac{\widehat{g}}{\log R+\gamma_{m}}
$$

approximates the harmonic measure of $C_{R}$ relative to the domain bounded only by $C_{R}$ and $A$, and is constant on $B$. Assume the approximation is exact. Then by Theorem 1 the extremal distance between $A$ and $C_{R}$ is a minimum, and by Theorem 2 it equals $\lambda_{R}^{b}$. But Theorem A and Green's theorem yield

$$
\frac{1}{\lambda_{R}^{b}}=D_{\Omega_{R}}\left(\frac{\widehat{g}}{\log R+\gamma_{m}}\right)=\frac{2 \pi}{\log R+\gamma_{m}},
$$

which gives (15). For an actual proof we must remove the assumptions that the approximation of the harmonic measure by $\widehat{g}$ is exact and that the domain $\Omega$ is extremal. 
Proof of Theorem 4. Suppose first that $\Omega$ is chosen to maximize the capacity of $A$. That is, assume that

$$
d(A)=d_{m}(A)=e^{-\gamma_{m}}
$$

where

$$
\gamma_{m}=\lim _{z \rightarrow \infty}\{\widehat{g}(z)-\log |z|\}
$$

Then for any $\epsilon>0$, there exists $R_{0}>0$ such that when $|z|>R_{0}$,

$$
|\widehat{g}(z)-\log | z\left|-\gamma_{m}\right| \leq \log (1+\epsilon) .
$$

Thus if $|z|=R /(1+\epsilon)>R_{0}$, then $\widehat{g}(z) \leq \log R+\gamma_{m}$. And if $|z|=R(1+\epsilon)$, then $\hat{g}(z) \geq \log R+\gamma_{m}$. Thus by the maximum principle for harmonic functions, the level curve $L_{R}=\left\{z: \hat{g}(z)=\log R+\gamma_{m}\right\}$ must lie between the circles $C_{R /(1+\epsilon)}$ and $C_{R(1+\epsilon)}$, as long as $R /(1+\epsilon)>R_{0}$. Let $O_{R} \subset \Omega$ be the domain bounded by $A, B$, and $L_{R}$, and let $\widehat{O}_{R}$ be the domain bounded only by $A$ and $L_{R}$. Since $\Omega$ is extremal, $\hat{g}$ is constant on the components of $B$. Thus the function

$$
\frac{\widehat{g}(z)}{\log R+\gamma_{m}}
$$

which takes the values 1 on $L_{R}$ and 0 on $A$, is the harmonic measure of $L_{R}$ relative to $\widehat{O}_{R}$ and is constant on the components of $B$. By Theorems 1 and 2, the extremal distance between $A$ and $L_{R}$ in $\widehat{O}_{R}$ equals the bridged extremal distance between $A$ and $L_{R}$ in $O_{R}$, using $B$ as the bridge:

$$
\lambda_{\widehat{O}_{R}}\left(A, L_{R}\right)=\lambda_{O_{R}}^{b}\left(A, B, L_{R}\right) .
$$

Since $L_{R}$ is bounded by $C_{R /(1+\epsilon)}$ and $C_{R(1+\epsilon)}$ for $R$ large,

$$
\lambda_{R /(1+\epsilon)}^{b} \leq \lambda_{O_{R}}^{b}\left(A, B, L_{R}\right) \leq \lambda_{R(1+\epsilon)}^{b},
$$

by the comparison principle for extremal length. But by Theorem A and Green's theorem,

$$
\frac{1}{\lambda_{\widehat{O}_{R}}\left(A, L_{R}\right)}=D_{\widehat{O}_{R}}\left(\frac{\widehat{g}}{\log R+\gamma_{m}}\right)=\frac{2 \pi}{\log R+\gamma_{m}} .
$$

Combine these last two results to see that

$$
\lambda_{R /(1+\epsilon)}^{b}-\frac{1}{2 \pi} \log R \leq \frac{1}{2 \pi} \gamma_{m} \leq \lambda_{R(1+\epsilon)}^{b}-\frac{1}{2 \pi} \log R
$$


whenever $R$ is large. If we now add $\frac{1}{2 \pi} \log (1+\epsilon)$ to both sides of the left-hand inequality in (17), we get

$$
\lambda_{R /(1+\epsilon)}^{b}-\frac{1}{2 \pi} \log \left(\frac{R}{1+\epsilon}\right) \leq \frac{1}{2 \pi}\left(\gamma_{m}+\log (1+\epsilon)\right),
$$

whence

$$
\limsup _{R \rightarrow \infty}\left\{\lambda_{R}^{b}-\frac{1}{2 \pi} \log R\right\} \leq \frac{1}{2 \pi}\left(\gamma_{m}+\log (1+\epsilon)\right)
$$

Since $\epsilon>0$ is arbitrary,

$$
\limsup _{R \rightarrow \infty}\left\{\lambda_{R}^{b}-\frac{1}{2 \pi} \log R\right\} \leq \frac{1}{2 \pi} \gamma_{m}
$$

A similar manipulation of the right-hand inequality in (17) yields

$$
\liminf _{R \rightarrow \infty}\left\{\lambda_{R}^{b}-\frac{1}{2 \pi} \log R\right\} \geq \frac{1}{2 \pi} \gamma_{m}
$$

This proves (15) if $\Omega$ is extremal for maximizing the capacity of $A$.

What if $\Omega$ is not extremal? The limit in (15) is invariant under any normalized conformal map of $\Omega$. The same normalized invariance for reduced extremal distance was noted by Ahlfors and Beurling in [3]. The proof (see [12], Ch. 2, Sec. 23) easily adapts to our case. Since by Theorem 1 there is some normalized conformal map of $\Omega$ which maximizes the capacity of $A$, our proof is complete.

For fixed $R$, let $\mathbf{b}_{R}=\left(b_{1}(R), \ldots, b_{m}(R)\right), \mathbf{w}_{R}=\left(w_{1}(R), \ldots, w_{m}(R)\right)$, and $\mathbf{Q}_{R}=\left(q_{i j}(R)\right)$ be the constants from Theorem 1 defining the minimal extremal distance between $A$ and $C_{R}$ in $\Omega_{R}$. Thus $\mathbf{Q}_{R} \mathbf{b}_{R}^{T}=\mathbf{w}_{R}^{T}$. In view of Theorem 4 , it is natural to expect a corresponding relation between these constants and the constants given by $\mathbf{P c}^{T}=\mathbf{v}^{T}$ from Theorem B defining maximal capacity in $\Omega$. The following theorem, which we state without proof, describes the connection. Assertions (i) and (ii) describe the asymptotic behavior of the period matrix associated with the harmonic measures of $\Omega_{R}$ as the circle $C_{R}$ is dilated to infinity. The example following the theorem provides a geometric interpretation of the constants $b_{i}(R)$ and $c_{i}$, and illustrates assertion (iii).

Theorem 5. With the above definitions,

(i) $\lim _{R \rightarrow \infty} \mathbf{Q}_{R}=\mathbf{P}$ 
(ii) $\lim _{R \rightarrow \infty}(\log R) \mathbf{w}_{R}=\mathbf{v}$

(iii) $\lim _{R \rightarrow \infty}(\log R) \mathbf{b}_{R}=\mathbf{c}$

Example. Suppose that $A$ is a single component of $\Gamma$. Then there is a normalized conformal map $F$ of $\Omega$ such that $F(\Omega)$ is the exterior of a disk centered at the origin minus concentric circular slits outside the disk. The map may be chosen so that $F(A)$ is the boundary of the disk, and each component of $F(B)$ is one of the arcs. Relabel $F(\Omega)$ to be $\Omega$. Then the capacity of $A$ is the radius $r$ of the disk. Green's function $\widehat{g}$ of the exterior of $A$ is $\log (|z| / r)$, and is constant on each component of $B$. Thus by Theorem $\mathrm{B}$, the capacity of $A$ is maximum, so $\gamma_{m}=-\log r$. Let $r_{i}$ be the radius of the $\operatorname{arc} B_{i}$. Then $c_{i}=\widehat{g}\left(B_{i}\right)=\log \left(r_{i} / r\right)$.

Let $R$ be large enough so that $C_{R}$ encloses all of $\Gamma$. Then $\Omega_{R}$ is a slit annulus. As in the example in Section $3, \lambda_{R}^{b}=\frac{1}{2 \pi} \log R / r$, so

$$
\lambda_{R}^{b}-\frac{1}{2 \pi} \log R=-\frac{1}{2 \pi} \log r=\frac{1}{2 \pi} \gamma_{m},
$$

which confirms Theorem 4. Since $\widehat{\eta}_{R}(z)=\log (|z| / r) / \log (R / r)$, we have $b_{i}(R)=\widehat{\eta}_{R}\left(B_{i}\right)=\log \left(r_{i} / r\right) / \log (R / r)$. Thus

$$
(\log R) b_{i}(R)=\frac{\log R \log \frac{r_{i}}{r}}{\log R-\log r}
$$

which converges to $\log \frac{r_{i}}{r}=c_{i}$ as $R$ approaches infinity. This verifies statement (iii) of Theorem 5.

\section{Maximal extremal distance and Robin capacity.}

In Theorem 1 we considered the problem of minimizing the extremal distance between the subsets $X$ and $Z$ on the boundary of the domain $G$. We now discuss the opposite problem of maximal extremal distance and relate it to Robin capacity.

For simplicity, we assume that each component of $S=\partial G$ is an analytic Jordan curve. For any conformal map $f$ of $G$, we defined $\widehat{f(G)}$ to be the domain containing $f(G)$ bounded only by $f(X)$ and $f(Z)$, and $\widehat{\lambda}_{f}$ to be the extremal distance between $f(X)$ and $f(Z)$ in $\widehat{f(G)}$. Define now the maximal extremal distance between $X$ and $Z$ to be

$$
\widehat{\Lambda}=\sup _{f} \hat{\lambda}_{f}
$$

where the supremum is taken over all conformal maps $f$ of $G$. In analogy to Theorems 1 and 2 for minimal extremal distance, the next theorem computes 
maximal extremal distance by a Dirichlet integral and characterizes extremal domains . For a given map $f$, recall that $\widehat{\eta}_{Z}$ is the harmonic measure of $f(Z)$ relative to $\widehat{f(G)}$.

Theorem 6. Let $\lambda_{G}(X, Z)$ denote the extremal distance between $X$ and $Z$ in $G$; that is, the extremal length of the family of curves in $G$ connecting $X$ and $Z$. Then

$$
\widehat{\Lambda}=\lambda_{G}(X, Z)=D_{G}(w),
$$

where $w(z)$ is the unique harmonic function in $G$ such that $w(z) \equiv 1$ on $Z$, $w(z) \equiv 0$ on $X$, and $\frac{\partial w}{\partial n}=0$ on $Y$. Extremal functions $F$ satisfying $\widehat{\lambda}_{F}=\widehat{\Lambda}$ always exist. Extremal domains $F(G)$ are characterized by the property that each component $F\left(Y_{i}\right)$ of $F(Y)$ is a union of analytic arcs on which

$$
\frac{\partial \widehat{\eta}_{Z}}{\partial n}=0
$$

Sketch of proof. The proof of existence of extremal domains is exactly as in Theorem 1. The proof of the characterization of extremal domains proceeds also as in Theorem 1, through the method of boundary variation. If $G$ maximizes the extremal distance between $X$ and $Z$, Schiffer's theorem tells us that $Y$ is the union of analytic arcs lying on the trajectories of the quadratic differential

$$
H_{Z}^{\prime}(z)^{2} d z^{2}>0
$$

where $H_{Z}$ is the analytic completion of $\widehat{\eta}_{Z}$ in $\widehat{G}$. This leads us to conclude that

$$
\frac{d}{d t} \operatorname{Im}\left\{H_{Z}(z(t))\right\}=0
$$

on $Y$, where $z(t)$ is a parametrization of any component of $Y$. By the Cauchy-Riemann equations, this implies that

$$
\frac{\partial \widehat{\eta}_{Z}}{\partial n}=\frac{\partial}{\partial n} \operatorname{Re}\left\{H_{Z}\right\}=0
$$

on $Y$. To compute $\widehat{\Lambda}$, observe as in (6) that

$$
\frac{1}{\widehat{\Lambda}}=D_{\widehat{G}}\left(\widehat{\eta}_{Z}\right)=\int_{\partial \widehat{G}} \widehat{\eta}_{Z} \frac{\partial \widehat{\eta}_{Z}}{\partial n} d s=\int_{Z} \frac{\partial \widehat{\eta}_{Z}}{\partial n} d s
$$

Let $w(z)$ be as in the statement of the theorem. Then $w$ and $\widehat{\eta}_{Z}$ have the same mixed boundary values on $S=\partial G$, so must agree in $G$. In particular,

$$
\int_{Z} \frac{\partial \widehat{\eta}_{Z}}{\partial n} d s=\int_{Z} \frac{\partial w}{\partial n} d s
$$


But

$$
\int_{Z} \frac{\partial w}{\partial n} d s=\int_{\partial G} w \frac{\partial w}{\partial n} d s=D_{G}(w)=\frac{1}{\lambda_{G}(X, Z)},
$$

by Theorem A.

We have shown that if $G$ is an extremal domain, maximizing the extremal distance between $X$ and $Z$ in $\widehat{G}$, then $\frac{\partial \widehat{\eta}_{Z}}{\partial n}=0$ on $Y$. But as in Theorem 1 , if $\widehat{\eta}_{Z}$ has this property, the above calculations lead to the same extremal distance, which is thus maximum. The property therefore characterizes extremal domains.

By way of illustration, suppose $X$ and $Z$ are single components of $S$. There is some conformal map $F_{1}$ such that the extremal distance between $F_{1}(X)$ and $F_{1}(Z)$ is maximum. If we follow by a map $F_{2}$ of $\widehat{F_{1}(G)}$ onto an annulus centered at the origin, then the extremal distance between $F_{2} \circ F_{1}(X)$ and $F_{2} \circ F_{1}(Z)$ is still a maximum, and $F_{2} \circ F_{1}(G)$ is an annulus with some pieces (corresponding to $F_{2} \circ F_{1}(Y)$ ) removed. Call the new domain $D$, and call the corresponding boundary subsets $\widetilde{X}, \widetilde{Y}$, and $\widetilde{Z}$. Then $\widehat{D}$ is an annulus, say of inner radius 1 and outer radius $P$, so $\widehat{\eta}_{Z}(z)=\log |z| / \log P$. The curves in $\widehat{D}$ on which $\frac{\partial \widehat{\eta}_{Z}}{\partial n}=0$ are exactly the radial segments connecting $\widetilde{X}$ and $\widetilde{Z}$, so by the characterization of extremal domains, each component of $\tilde{Y}$ must lie on a radial segment. We thus have an independent proof of the existence and uniqueness of the well-known map of a multiply-connected domain onto a radially-slit annulus. The extremal distance $\lambda_{G}(X, Z)$ equals the maximal extremal distance $\hat{\lambda}$, which is $\frac{1}{2 \pi} \log P$. Thus, for any conformal map $f$ of $G$, the modulus of the doubly-connected domain bounded by $f(X)$ and $f(Z)$ is bounded from above by $P$.

Return now to the setting for maximal capacity, where $\Omega$ is a finitelyconnected domain containing infinity. We showed in the proof of Theorem 4 that a map maximizing the capacity of the boundary subset $A$ must essentially minimize the extremal distance between $A$ and the circles $C_{R}$ for large $R$. This is reflected in the asymptotic relation

$$
\frac{1}{2 \pi} \gamma_{m}(A)=\lim _{R \rightarrow \infty}\left\{\lambda_{R}^{b}-\frac{1}{2 \pi} \log R\right\},
$$

where we recognized the bridged extremal distance $\lambda_{R}^{b}$ as the minimal extremal distance between $A$ and $C_{R}$.

Recall that Robin's function $R(z)$ is the unique function harmonic in $\Omega \backslash \infty$ such that $R(z) \equiv 0$ on $A$, the normal derivative $\frac{\partial R}{\partial n}$ is zero on $B$, and such that $R(z)-\log |z|$ is harmonic at infinity. The Robin capacity of $A$ relative to $\Omega$ is $\delta(A)=e^{-\rho(A)}$, where

$$
\rho(A)=\lim _{z \rightarrow \infty}\{R(z)-\log |z|\} .
$$


Duren and Schiffer [6] showed that Robin capacity can be interpreted as minimal capacity:

$$
\delta(A)=\inf _{f} d(f(A)),
$$

where the infimum ranges over all normalized conformal maps of $\Omega$ preserving infinity. In [5] Duren and Pfaltzgraff exploited the following relation between extremal length and Robin capacity:

$$
\frac{1}{2 \pi} \rho(A)=\lim _{R \rightarrow \infty}\left\{\lambda_{\Omega_{R}}\left(A, C_{R}\right)-\frac{1}{2 \pi} \log R\right\} .
$$

Since we can now recognize $\lambda_{\Omega_{R}}\left(A, C_{R}\right)$ as the maximal extremal distance between $A$ and $C_{R}$, equation (18) suggests that maps minimizing the capacity of $A$ essentially maximize the extremal distance between $A$ and $C_{R}$. As in the proof of Theorem 4, we could make this more rigorous by exploiting the similar characterizations for maximal extremal distance and Robin capacity. Duren and Schiffer showed that $d(f(A))$ is minimum if and only if Green's function $\widehat{g}$ of the exterior of $f(A)$ satisfies $\frac{\partial \widehat{g}}{\partial n} \equiv 0$ on $f(B)$.

\section{More general partitions of the boundary.}

Suppose $G$ is an annulus, and $X$ and $Y$ are the top and bottom halves, respectively, of the inner circle. For any conformal map $f$ of $G$, we set $f(X)=\{C(f, z): z \in X\}$, where $C(f, z)$ denotes the cluster set of $f$ at the point $z$. What can we say about the minimal and bridged extremal distances between $X$ and the outer circle $Z$ in this case? We briefly indicate below how the results on minimal extremal distance and bridged extremal distance might generalize to arbitrary partitions $X \cup Y \cup Z$ of the boundary $S$ of $G$.

In $[14]$ we found that the maximal capacity $d_{m}(A)$ for arbitrary partitions $A \cup B$ of the boundary $\Gamma$ equals $d_{m}([A])$, where $[A]$ is the union of all components with which $A$ has non-empty intersection. In other words, if even a single point of $A$ lies on a component of $\Gamma$, the maximal capacity is the same as if that entire component belonged to $A$. This basically follows from the fact that, with the cluster point definition for $f(A)$, we can find a conformal map $f$ such that $f(A)=f([A])$, no matter how thinly $A$ is distributed. The connection between maximal capacity and bridged extremal distance thus makes the following conjecture plausible.

Conjecture . Let $Z$ be a union of components of $S$, and let $X \cup Y$ be an arbitrary partition of $S \backslash Z$. Let $(Y)=Y \backslash[X]$. Then

$$
\widehat{\lambda}_{G}(X, Y, Z)=\widehat{\lambda}_{G}([X],(Y), Z)
$$


and

$$
\lambda_{G}^{b}(X, Y, Z)=\lambda_{G}^{b}([X],(Y), Z) .
$$

So, for example, the bridged extremal distance between the top half of the inner circle of the annulus and the outer circle should be the same as the extremal distance between the inner and outer circles.

The conjecture is easily proved if we include in the family of bridging curves from $X$ to $Z$ all paths from $[X]$ to $Z$. One can justify including these curves in the following way. Suppose a component $S_{i}$ of $S$ contains points of $X$ and $Y$. A path $\gamma$ from a point in $Y \cap S_{i}$ to $Z$ does not connect $X$ to $Z$. But let $p$ be any point in the non-empty set $\bar{X} \cap \bar{Y} \cap S_{i}$. The "path" $\{p\} \cup \gamma$ "bridges" $Y$ in connecting $X$ to $Z$, and has the same $\rho$-length as $\gamma$ for any admissible metric $\rho$.

If we instead insist that all bridging curves contain an initial segment in $G$ with endpoint in $X$, the assertion for bridged extremal distance is still true if the family of curves in $G$ connecting $X$ to $Y$ has zero extremal length. If it does not, the question remains open.

\section{Earlier work on bridged extremal distance.}

In [8] and [9] Minda extended results of Marden and Rodin [7] in computing a generalization of bridged extremal distance on Riemann surfaces. He considered partitions $X \cup Y \cup Z \cup W$ of the components of $S$, where the bridge $Y$ is further partitioned into subsets $Y_{i}, i=1, \ldots, n$, each $Y_{i}$ a union of components of $S$. A curve connecting $X$ to $Z$ in his bridging family is allowed to stop at any $Y_{i}$ and re-emerge from a point on any other component of $Y_{i}$. Minda computes the extremal length of this family of curves with the Dirichlet integral of a harmonic function $u$, uniquely defined by the following conditions: $u \equiv 0$ on $X, u \equiv 1$ on $Y, \frac{\partial u}{\partial n} \equiv 0$ on $W$, and $u \equiv b_{i}$ on $Y_{i}$, where the constants $\left\{b_{i}\right\}$ are uniquely determined by requiring $\int_{Y_{i}} \frac{\partial u}{\partial n} d s=0$ for $i=1, \ldots n$ (compare with Theorems 1, 2 and Theorem 3 , assertion (ii)). He does not compute the $b_{i}$ explicitly. Aside from our more restrictive definition, the point of departure in this paper has been to connect bridged extremal distance to the extremal problems of minimal extremal distance and maximal capacity. Minda uses bridged extremal distance to devise tests for degeneracy in the classification of Riemann surfaces [8], and to give extremal length interpretations of reproducing differentials on Riemann surfaces [9]. 
[1] L. Ahlfors, Complex Analysis, 3rd ed., McGraw-Hill, New York, 1979.

[2] , Conformal invariants: topics in geometric function theory, McGraw-Hill, New York, 1973.

[3] L. Ahlfors and A. Beurling, Conformal invariants, Construction and Applications of Conformal Maps, Proceedings of a Symposium, (E.F. Beckenbach, Ed.), National Bureau of Standards, Appl. Math. U. S. Government Printing Office, Washington, DC, 18 (1952), 243-245.

[4] P. Duren, Univalent functions, Springer-Verlag, New York, 1983.

[5] P. Duren and J. Pfaltzgraff, Robin capacity and extremal length, J. Math. Anal. Appl., 179 (1993), 110-119.

[6] P. Duren and M. M. Schiffer, Robin functions and distortion of capacity under conformal mapping, Complex Variables Theory Appl., 21 (1993), 189-196.

[7] A. Marden and B. Rodin, Extremal and conjugate extremal distance on open Riemann surfaces with applications to circular-radial slit mappings, Acta Math., 115 (1966), 237-269.

[8] C.D. Minda, Extremal length and harmonic functions on Riemann surfaces, Trans. Amer. Math. Soc., 171 (1972), 1-22.

[9] _ _ Extremal length and reproducing differentials on Riemann surfaces, J. Analyse Math., 29 (1976), 154-202.

[10] V.K. Navoyan, Continuity of the conformal capacity of a space condenser, Ukrain. Mat. Ž., 33 (1981), 421-426 (in Russian) = Ukrainian Math. J., 33 (1981), 325-329.

[11] Z. Nehari, Conformal mapping, McGraw-Hill, New York, 1952.

[12] M. Ohtsuka, Dirichlet problem, extremal length, and prime ends, Van Nostrand, New York, 1970.

[13] M.M. Schiffer, Hadamard's formula and variation of domain-functions, Amer. J. Math., 68 (1946), 417-448.

[14] R. Thurman, Upper bound for distortion of capacity under conformal mapping, Trans. Amer. Math. Soc., 346(2) (1994), 605-616.

Received November 1, 1994.

The Geometry Center

UNIVERSITY OF MiNNESOTA

MinNEAPOLIS, MN 55454

E-mail address: thurman@geom.umn.edu 


\title{
PACIFIC JOURNAL OF MATHEMATICS
}

\author{
Founded in 1951 by
}

\author{
$\begin{array}{ll}\text { E. F. Beckenbach (1906-1982) } & \text { F. Wolf (1904-1989) }\end{array}$
}

\section{EDITORS}

Sun-Yung A. Chang (Managing Editor) Robert Finn University of California

Los Angeles, CA 90095-1555

pacific@math.ucla.edu

\section{F. Michael Christ}

University of California

Los Angeles, CA 90095-1555

christ@math.ucla.edu

Nicholas Ercolani

University of Arizona

Tucson, AZ 85721

ercolani@math.arizona.edu
Stanford University

Stanford, CA 94305

finn@gauss.stanford.edu

Steven Kerckhoff

Stanford University

Stanford, CA 94305

spk@gauss.stanford.edu

Martin Scharlemann

University of California

Santa Barbara, CA 93106

mgscharl@math.ucsb.edu

\section{Gang Tian}

Massachusettes Institute of Technology

Cambridge, MA 02139

tian@math.mit.edu

\section{S. Varadarajan University of California Los Angeles, CA 90095-1555 vsv@math.ucla.edu \\ Dan Voiculescu \\ University of California \\ Berkeley, CA 94720 \\ dvv@math.berkeley.edu}

\section{SUPPORTING INSTITUTIONS}

ACADEMIA SINICA, TAIPEI

CALIF. INST. OF TECHNOLOGY

CHINESE UNIV. OF HONG KONG

HONG KONG UNIV. OF SCI. \& TECH.

KEIO UNIVERSITY

MACQUARIE UNIVERSITY

MATH. SCI. RESEARCH INSTITUTE

NEW MEXICO STATE UNIV.

OREGON STATE UNIV.

PEKING UNIVERSITY

RITSUMEIKAN UNIVERSITY

STANFORD UNIVERSITY

\author{
TOKYO INSTITUTE OF TECHNOLOGY \\ UNIVERSIDAD DE LOS ANDES \\ UNIV. OF ARIZONA \\ UNIV. OF BRITISH COLUMBIA \\ UNIV. OF CALIF., BERKELEY \\ UNIV. OF CALIF., DAVIS \\ UNIV. OF CALIF., IRVINE \\ UNIV. OF CALIF., LOS ANGELES \\ UNIV. OF CALIF., RIVERSIDE \\ UNIV. OF CALIF., SAN DIEGO \\ UNIV. OF CALIF., SANTA BARBARA
}

UNIV. OF CALIF., SANTA CRUZ

UNIV. OF HAWAII

UNIV. OF MELBOURNE

UNIV. OF MONTANA

UNIV. NACIONAL AUTONOMA DE MEXICO

UNIV. OF NEVADA, RENO

UNIV. OF OREGON

UNIV. OF SOUTHERN CALIFORNIA

UNIV. OF UTAH

UNIV. OF WASHINGTON

WASHINGTON STATE UNIVERSITY

The supporting Institutions listed above contribute to the cost of publication of this Journal, but they are not owners or publishers and have no responsibility for its contents or policies.

Manuscripts must be prepared in accordance with the instructions provided on the inside back cover.

The table of contents and the abstracts of the papers in the current issue, as well as other information about the Pacific Journal of Mathematics, may be found on the Internet at http://www.math.uci.edu/pjm.html.

The Pacific Journal of Mathematics (ISSN 0030-8730) is published monthly except for July and August. Regular subscription rate: $\$ 245.00$ a year (10 issues). Special rate: $\$ 123.00$ a year to individual members of supporting institutions.

Subscriptions, back issues published within the last three years and changes of subscribers address should be sent to Pacific Journal of Mathematics, P.O. Box 4163, Berkeley, CA 94704-0163, U.S.A. Prior back issues are obtainable from Kraus Periodicals Co., Route 100, Millwood, NY 10546.

The Pacific Journal of Mathematics at the University of California, c/o Department of Mathematics, 981 Evans Hall, Berkeley, CA 94720 (ISSN 0030-8730) is published monthly except for July and August. Second-class postage paid at Berkeley, CA 94704, and additional mailing offices. POSTMASTER: send address changes to Pacific Journal of Mathematics, P.O. Box 6143, Berkeley, CA 94704-0163.

\section{PUBLISHED BY PACIFIC JOURNAL OF MATHEMATICS at University of California,} Berkeley, CA 94720, A NON-PROFIT CORPORATION

This publication was typeset using AMS-LATEX,

the American Mathematical Society's TEX macro system.

Copyright (C) 1995 by Pacific Journal of Mathematics 


\section{PACIFIC JOURNAL OF MATHEMATICS}

\section{Volume $176 \quad$ No. $2 \quad$ December 1996}

One remark on polynomials in two variables

ENRIQUe ARTAl BARTOLO and PIERRETTE CASSOU-NOGUÈS

Divergence of the normalization for real Lagrangian surfaces near complex tangents XIANGHONG GONG

Classification of the stable homotopy types of stunted lens spaces for an odd prime JESUS GONZALEZ

Plancherel formulae for non-symmetric polar homogeneous spaces

JING-SONG HUANG

A uniqueness theorem for the minimal surface equation

JENN-FANG HWANG

Differential Galois groups of confluent generalized hypergeometric equations: an approach

using Stokes multipliers

Claudine Mitschi

Oscillatory theorem and pendent liquid drops

KIMIAKI NARUKAWA and TAKASHI SUZUKI

Local and global plurisubharmonic defining functions

ALAN NOELL

Specializations and a local homeomorphism theorem for real Riemann surfaces of rings

M. J. DE LA PUENTE

Eigenvalue comparisons in graph theory

GREGORY T. QUENELL

Applications of loop groups and standard modules to Jacobians and theta functions of isospectral curves

WILLI SCHWARZ

Bridged extremal distance and maximal capacity

ROBERT E. THURMAN

Imbedding and multiplier theorems for discrete Littlewood-Paley spaces

IGOR E. VERBITSKY

On constrained extrema

THOMAS VOGEL

Heat flow of equivariant harmonic maps from $\mathbb{B}^{3}$ into $\mathbb{C P} \mathbb{P}^{2}$

YUANLONG XIN

Proof of Longuerre's theorem and its extensions by the method of polar coordinates

ZHIHONG YU

Correction to: "Special generating sets of purely inseparable extension fields of unbounded exponent"

BONIFACE IHEMOTUONYE EKE 